\title{
Restored CD8+PD-1+ T cells Facilitate the Response to Anti-PD-1 for Patients with Pancreatic Ductal Adenocarcinoma
}

\section{Qian Zhu}

Wuhan University Zhongnan Hospital

guoliang qiao ( $\sim$ gqiao1@mgh.harvard.edu )

Harvard Medical School

\section{Lefu Huang}

Capital Medical University Affiliated Beijing Shijitan Hospital

\section{Chang Xu}

Second Military Medical University First Hospital: Changhai Hospital

\section{Deliang Guo}

zhongnan hospital

\section{Shuo Wang}

shijitan hospital

Jing Zhao

huazhong university

\section{Yuguang Song}

shijitan hospital

\section{Bing Liu}

chinese liberty hospital

\section{Zheng Chen}

capital medical university

\section{Zhiyong Yang}

Wuhan University Zhongnan Hospital

\section{Yufeng Yuan}

Wuhan University Zhongnan Hospital

\section{Research}

Keywords: CD8+PD-1+ T cell, TCR, PDAC

Posted Date: November 15th, 2021

DOI: https://doi.org/10.21203/rs.3.rs-1061490/v1 
License: (c) (i) This work is licensed under a Creative Commons Attribution 4.0 International License. Read Full License

Version of Record: A version of this preprint was published at Frontiers in Oncology on April 11th, 2022. See the published version at https://doi.org/10.3389/fonc.2022.837560. 


\section{Abstract}

Backgrounds

We aimed at to investigate that restoring the amount of $\mathrm{CD} 8^{+} \mathrm{PD} 1^{+} \mathrm{T}$ cells through adoptive $\mathrm{T}$ cell therapy (ACT) could improve the prognosis and facilitate the therapeutic response to anti-PD-1 in patients with advanced pancreatic cancer (APC).

Methods

177 adult patients who underwent tumor resection as initial treatment for PDAC during February 2013 to July 2019. at Zhongnan Hospital of Wuhan University were enrolled in this study. Another cohort of 32 patients with APC were prospectively enrolled from Capital Medical University Cancer Center, Beijing Shijitan Hospital from June 1, 2013, to May 30, 2019. All patients with APC underwent ACT and 15 of 32 (46.8\%) patients received ACT combined with anti-PD-1 (Pembrolizumab).

Results

Of the 177 patients received tumor resection, 67 tumor samples showed overexpression of PD-L1 We found that high PD-L1 expression in tumor tissues was significantly associated with short overall survival. Also, we tested the percentage of peripheral CD $8^{+} \mathrm{PD}-1^{+} \mathrm{T}$ cells and found it was significantly correlated with the PD-L1 expression and the prognosis of patients with PDAC. We further tracked the peripheral blood $T$ lymphocyte subtypes for 30 months and found that CD $8{ }^{+} P D-1^{+}$cells were decreased and we hypothesized that the $\mathrm{CD} 8^{+} \mathrm{PD}-1^{+}$cells were exhausted. After that, we performed ACT for patients with APC and we found that the ratios of post treatment of ACT/pre-ACT CD $8^{+} \mathrm{PD}-1^{+} \mathrm{T}$ cells were significantly related with the prognosis of patients with APC. Moreover, patients with combined treatment of ACT with anti-PD-1 had significantly favorable both OS and PFS. Furthermore, T-cell receptor (TCR) repertoire were tested and TCR diversity of cultured T cells were calculated and we found the treatment of ACT impacted on the TCR repertoire especially in patients with significantly CD $8^{+} \mathrm{PD}-1^{+} \mathrm{T}$ cells enhanced.

\section{Conclusions}

this study showed that the CD $8^{+} \mathrm{PD}-1^{+} \mathrm{T}$ cell subgroup was related with expression of PD-L1 and the prognosis of patients with PDAC who received surgical resection. The CD $8^{+} P D-1^{+} T$ cells were gradually exhausted and restoring it by treatment of ACT was associated with a significantly favorable prognosis and facilitate the response to Anti-PD-1.

\section{Background}

Pancreatic Ductal Adenocarcinoma (PDAC) is one of the most aggressive solid malignancies and the leading cause of cancer-related deaths. Surgical resection is the main radical treatment but less than $20 \%$ of patients have a resectable tumor at the time of diagnosis ${ }^{1,2}$. For PDAC patients with metastatic 
disease, the treatment remained challenging and the median survival was 6-12 months, despite the availability of multi-agents chemotherapy and radiotherapy ${ }^{3,4}$. Immunotherapy is among the most promising strategies under development but is complicated by the immunosuppressive tumor microenvironment prevalent in PDAC ${ }^{5,6}$. The prognosis has not given satisfactory results in patients with advanced pancreatic cancer (APC) who received immunotherapy ${ }^{7-9}$. Programmed death receptor 1 (PD1 ) is one of the most important checkpoint pathways and has been approved to be used in various cancers $^{10,11}$. Several investigations have showed anti-PD-1 were not effective in patients with PDAC since targeting this pathway should induce $T$ cell activity and consequently cancer cell death ${ }^{12}$. However, adoptive cell therapy (ACT), delivery of ex vivo activated cellular products such as dendritic (DC), NK, and/or T cells has shown activity in pancreatic cancer. Widespread use of ACT is enabled by practical generation processes, rapid expansion ex vivo, and $\mathrm{MHC}$-unrestricted tumor cell killing. Further, it is feasible to combine ACT with chemotherapy as we previously demonstrated in patients with APC ${ }^{13}$.

The ACT product is a complex mixture of cell types and we hypothesize that greater efficacy would be achieved by a better understanding of which cell subtypes affect clinical outcome so that they may be modulated ${ }^{14}$. We have observed an increase in $\mathrm{CD}^{+} \mathrm{T}$ cells that express programmed death 1 (PD-1, CD279) within expanded cellular products. PD- 1 expressed by $C D 4^{+}$and $C D 8^{+} T$ cells, is often viewed as a co-inhibitory receptor and induced by T-cell receptor(TCR) signaling, the engagement of which by its ligands PD-L1 and PD-L2 on tumors and other immune cells, impairs effector T cell function ${ }^{15}$. Previous studies showed that PD-1 expression levels in virus-specific, peripheral blood CD8 ${ }^{+}$T-cells correlated with disease progression of some viral infections in humans ${ }^{16-18}$. Tumor infiltrating lymphocytes (TILs) that express high levels of PD-1 are functionally impaired, failing to produce cytokines such as interleukin 2 19-22 and the presence of intratumoral PD-1 signaling has been associated with a worse survival in PDAC ${ }^{23}$. However, PD-1 is also a marker of activated T cells and may identify a population of $\mathrm{T}$ cells with potential antitumor activity. Rosenberg et al observed that $C D 8^{+} \mathrm{PD} 1^{+} \mathrm{TILs}$ recovered reactivity after exposure to high dose IL-2, resulting in higher tumor specific IFN-y production compared with CD $8^{+} \mathrm{PD} 1^{-}$ T cells ${ }^{22}$.

Therefore, in present study, we hypothesized that $\mathrm{CD} 8^{+} \mathrm{PD} 1^{+} \mathrm{T}$ cells were the cell subset that gradually exhausted after tumor resection in selected patients and restoring the amount of $\mathrm{CD} 8^{+} \mathrm{PD} 1^{+} \mathrm{T}$ cells through ACT could improve the prognosis and facilitate the therapeutic response to anti-PD-1 in patients with APC.

\section{Patients And Methods}

\section{Source of patients and clinical specimens.}

177 adult patients (age >18 years old) who underwent tumor resection as initial treatment for PDAC during February 2013 to July 2019. at Zhongnan Hospital of Wuhan University were enrolled in this study. The inclusion criteria were: (1) no anticancer treatments before enrollment; (2) no additional adjuvant 
chemotherapy routinely administered unless a recurrence was identified; (3) no other malignancies simultaneously. The exclusion criteria were: (1) repeat tumor resection; (2) presence of cardiac, pulmonary or renal insufficiency before operation. The patients' clinical data were retrospectively collected and included demographics, body-mass-index (BMI), and preoperative CA19-9 levels. Postoperative outcomes and treatment included the occurrence of major morbidity (Clavien-Dindo $\geq \mathrm{III}$ ) and 30-day mortality. Pathological parameters were collected according to the 8th edition of the AJCC TNM staging system and included tumor stage, tumor size, extent of lymph-node involvement, and tumor grading. Phenotypic analysis of peripheral blood immune cells was tested and followed up every month after surgery and other follow-up data were obtained from their most recent medical review, which consisted of a clinical examination and an assessment of computed tomography (CT) scans. Patients' overall survival (OS) time was calculated from the surgery date to the date of death or last contact. An independent biostatistician managed and maintained the collected data.

Another cohort of 32 patients with advance PDAC were enrolled from Capital Medical University Cancer Center, Beijing Shijitan Hospital from June 1, 2013, to May 30, 2019. The study was approved by the Regional Ethical Review Board for Capital Medical University Cancer Center. Patients were treated according to the Declaration of Helsinki's ethical principles for medical research involving human subjects. All patients provided an informed written consent prior to study entry. All patients underwent ACT and 15 of 32 (46.8\%) patients received ACT combined with anti-PD-1 (Pembrolizumab). Patients' OS time was calculated from the ACT treatment date to the date of death or last contact.

\section{Preparation of ACT product for sorting}

The ACT product was generated ex vivo as described in detail previously ${ }^{24}$. Peripheral blood stem cells were mobilized by injection of GM-CSF $5 \mathrm{mcg} / \mathrm{kg}$ per day (Chugai Pharm Co. Ltd., Japan) until the level of mononuclear cells in peripheral blood reached $1.5 \times 10^{9} / \mathrm{L}$. Then, PBMCs were collected by a COBE Spectra cell separator (COBE BCT, Lakewood, CO, USA) until the CD34+ count reached a threshold of $4.5 \times$ $10^{6} / \mathrm{kg}$. All collections were frozen at $-80^{\circ} \mathrm{C}$ until required for further analysis. Between $30-50 \mathrm{ml}$ of thawed apheresis product was co-cultured 7 days with IL-4 (1,000 U/mL; R\&D Systems, Inc., Minneapolis, MN), TNF-a (20 ng/mL; R\&D Systems, Inc., Minneapolis, MN) and GM-CSF (800 U/mL; Amoytop Biotech Co., Ltd., Xiamen, China) to generate autologous DCs. Another aliquot of PBMCs was expanded in complete medium consisting of AIM-V supplemented with $10 \%$ heat-inactivated human $A B$ serum and the recombinant cytokines IL-2 at 2,000 U/mL (Boehringer Mannheim, Germany) and CD3 antibody at 1.7 $\mathrm{mg} / \mathrm{mL}$ (Boehringer Mannheim, Germany). Subsequently, half of the media was replaced with fresh AIM$V$ containing IL-2 (2000IU/ml) every other day. After 7-10 days, the autologous DCs were mixed with cultured CTLs at a ratio of 1:100 for 7 days, and then the co-cultured DC-CTLs were harvested.

\section{Generation and sorting of CD8 ${ }^{+} \mathrm{PD}-1^{+} \mathrm{T}$ cells}

The $\mathrm{CD} 8^{+} \mathrm{PD}-1^{+} \mathrm{T}$ cells were sorted from the ex vivo expanded $\mathrm{T}$ cell products. Cell counts were recorded daily from day 0 to day 30 . The sorted CD $8^{+} P D-1^{+} T$ cells were tested for anti-tumor activities assay in 
vitro. The TCR repertoire of cultured cells was performed at day 0 and day 30 to determine the association with peripheral blood lymphocyte phenotype after ACT infusion and subsequent clinical response.

\section{Flow cytometric analysis and sorting}

We used the following fluorochrome-conjugated antibodies: CD3 PerCP-Cy5.5, CD4 FITC, CD8 FITC, CD25 PE, CD28 PE, CD 56 PE (Beckman), and PD-1 PE, LAG-3 PE, 4-1BB PE, TIM-3 PeCy-7 (Biolegend). We detected the cell subpopulation of PBMCs prior to culture and within cultured CTLs by flow cytometric analysis as described previously ${ }^{24}$. Briefly, cells were re-suspended in staining buffer and then stained with primary antibody at $4^{\circ} \mathrm{C}$ for $30 \mathrm{~min}$ in the dark. Stained cells were centrifuged for $10 \mathrm{~min}$ at 1,500 $\mathrm{rpm}$ at room temperature and subsequently washed in staining buffer twice prior to FACS analysis. Threecolor flow cytometric analysis was run to determine cell phenotypic using Cytomics FC500 and CXP analysis software (Beckman-Coulter USA). $\mathrm{CD} 8^{+} \mathrm{PD} 1^{+} \mathrm{T}$ cell sorting was carried out using the MoFlo Astrios EQ (Beckman, USA). first, CD8 ${ }^{+}$cells were enriched using CD8 microbeads (Biolegend) and the enriched T cells were incubated with FITC-conjugated-CD8, PC5.5-conjugated-CD3, and PE-conjugated$\mathrm{PD}-1$ at $4^{\circ} \mathrm{C}$ for $30 \mathrm{~min}$. Cell sorting were based on the gate strategy $\left(\mathrm{PI}^{-}, \mathrm{CD} 3^{+}, \mathrm{CD} 8^{+}\right.$and $\left.\mathrm{PD}-1^{+}\right)$. The sorted populations were expanded to detect their reactivity on day 13-15 with irradiated allogeneic feeder cells $(5,000 \mathrm{rad})$ pooled from three donors in T cell medium supplemented with $10 \%$ human AB serum, anti-CD3 and IL-2 (2,000 IU/ml) (Boehringer Mannheim, Germany).

\section{Assessment of tumor recognition and cytotoxicity assay}

IFN- $y$ enzyme-linked immunospot (ELISPOT) assay was used to measure recognition of targets. After 15 days of in vitro culture in cell medium supplemented with $2,000 \mathrm{IU} / \mathrm{ml} \mathrm{IL}-2$, at $37^{\circ} \mathrm{C}$ in $5 \% \mathrm{CO}_{2}$, cultured T cells were washed and co-cultured, either alone or with HLA-A2+ target tumor cell. In the ELISPOT assays, effector cells $\left(1 \times 10^{5}\right)$ were added to target cell lines $\left(1 \times 10^{4}\right)$ at an E:T ratio of 10:1 per well in a 96-well plate and incubated for $24 \mathrm{~h}$, according to the manufacturer's instructions. The raw data were analyzed and plotted using CTL Immunospot software (Cellular Technology Limited, USA). The identification of greater than 40 spots and twice background was required to report positive $T$ cell reactivity.

A Cell Counting Kit-8 (CCK-8) was used to detect cytolytic activity. Target cells were plated with effector cells at various effector/target ratios (6.25:1, 12.5:1, 25:1 and 50:1) in 96-well U-bottomed plate for 24 hours at $37^{\circ} \mathrm{C}$. The supernatants were harvested for absorbance measurement in a microplate reader at $450 \mathrm{~nm}$.

\section{7. $T$ cell receptor (TCR) sequencing}

DNA was extracted from ex vivo expanded T cells using a Qiagen DNA FFPE kit, DNA blood kit, or DNA blood mini kit (Qiagen). TCR Vb CDR3 sequencing was performed using the survey (cultured cells) or deep (PBMC) resolution Immunoseq platforms. Bio-informatic and bio-statistical analyses of productive 
clones were performed to assess the dynamics of expanded T cells. The TCR Vb CDR3 sequence diversity at day 15 during the expansion was compared to the initial TCR diversity.

\section{Phenotypic analysis of peripheral blood immune cells}

The technique protocol was similar with our previous reports. Peripheral venous blood was obtained from each patient at various time points after ACT infusion. Whole blood $(100 \mu \mathrm{l})$ was incubated in the dark with primary antibody at $4^{\circ} \mathrm{C}$ for $15 \mathrm{~min}$. Anti-CD3-FITC/anti-CD56-RPE (Dako), anti-CD3-FITC (fluorescein isothiocyanate), anti-CD4-RPE, anti-CD8-RPE, anti-CD45RO, and anti-CD4-FITC/anti-CD25-PE (BD Bioscience) were used. After hemolysis for $10 \mathrm{~min}$, samples were centrifuged for $10 \mathrm{~min}$ at 1,500 rpm at room temperature, and then washed twice in PBS and subjected to flow cytometric analysis. Threecolor flow cytometric analysis was performed to determine cell phenotypes using an FC500 (BeckmanCoulter), and CXP analysis software (Beckman-Coulter). Lymphocytes were gated by forward scatter versus side scatter. Analysis was set to collect 5,000 gated events.

\section{Statistical methods.}

Continuous variables were expressed as mean \pm SD (standard deviation) and compared using a twotailed unpaired Student's t test; categorical variables were compared using c2 or Fisher analysis. Lifetable estimates of survival time were calculated for the evaluation of PFS and OS as the primary endpoint, according to the Kaplan and Meier methodology ${ }^{25}$. ROC (Receiver Operating Characteristic) curves were used to confirm the cut-off values of post/pre CD $8^{+} \mathrm{PD}-1^{+}, \mathrm{CD} 8^{+} \mathrm{LAG}-3^{+}, \mathrm{CD} 8^{+} \mathrm{TIM}-3^{+} \mathrm{T}$ cells, post/pre Shannon index, Clonality, Evenness and post/pre TCR subclones. All statistical evaluations were carried out using SPSS software (Statistical Package for the Social Science, version 15.0, SPSS Inc, Chicago, IL) and GraphPad Prism 5 (Version 5.01, GraphPad Software, Inc., USA). A value of $p<0.05$ was considered to be statistically significant in all the analyses.

\section{Results}

\section{Patient characteristics}

A total of 177 patients with PDAC who underwent surgical resection in Zhongnan Hospital of Wuhan University from February 2013 to July 2019. Patients were divided into two groups including PD-L1 expression high $(n=67)$ and PD-L1 expression low $(n=110)$. Characteristics of all patients are detailed in Table 1. There were no significant differences in BMI, estimated blood loss, hospital length of stay, and the proportion of R0 resection between these two groups. Concurrently, 32 patients with advanced PDAC were enrolled in this study at the Capital Medical University Cancer Center, Beijing Shijitan Hospital from June 1, 2013, to May 30, 2019. Characteristics of all patients are detailed in Table 2. The majority had metastatic disease and multiple sites of disease, and $56 \%$ were PS 2. 
Table 1

Demographics and Baseline Characteristics of Patients with PDAC who underwent tumor resection.

\section{Variable}

High-expression of PDL-1

(67)
Low-expression of PDL-1

(110)

\section{Sex}

Female

28

50

Male

39

60

Age(years)

$57.6(35-75)$

$56.8(38-80)$

ASA Score classification

2

30

58

3

37

52

BMl kg/m2

$24.2 \pm 4.7$

$25.1 \pm 5.4$

Pre-operation Serum CA-199 U/ml

$<37$

20

69

$\geq 37$

47

41

Tumor and pathologic

characteristics

\section{AJCC TNM stage}

\begin{tabular}{lll} 
I & 11 & 23 \\
III & 35 & 78 \\
Grade & 21 & 9 \\
\hline G1 & & \\
G2 & 5 & 12 \\
G3 & 21 & 48 \\
G4 & 32 & 33 \\
Tumor size(cm) & 9 & 17 \\
Neural Invasion & $2.32 \pm 1.65$ & $2.53 \pm 1.77$ \\
yes & & 43 \\
no & 25 & 67 \\
\hline Vascular invasion & 42 & \\
\hline
\end{tabular}




\begin{tabular}{|lll|}
\hline Variable & $\begin{array}{l}\text { High-expression of PDL-1 } \\
(\mathbf{6 7 )}\end{array}$ & $\begin{array}{l}\text { Low-expression of PDL-1 } \\
(\mathbf{1 1 0})\end{array}$ \\
\hline yes & 45 & 42 \\
\hline no & 22 & 68 \\
\hline Nodal status: ypN & & 57 \\
\hline 0 & 17 & 39 \\
\hline 1 & 38 & 14 \\
\hline 2 & 12 & 77 \\
\hline Tumor resection & & 33 \\
\hline R0 & 52 & \\
\hline R1 & 15 & 92 \\
\hline Operation type & & 18 \\
\hline Whipple & 60 & 77 \\
\hline distal pancreatectomy & 7 & \\
\hline
\end{tabular}


Table 2

Demographics and Baseline

Characteristics of Patients with APC.

\begin{tabular}{|ll|}
\hline Variable & Median/Number \\
\hline Total enrollment & 32 \\
\hline age & $60.4 \pm 6.1$ \\
\hline Sex & \\
\hline Female & 15 \\
\hline Male & 17 \\
\hline ECOG-PS & \\
\hline 1 & 11 \\
\hline 2 & 21 \\
\hline TNM staging & \\
\hline III & 4 \\
\hline IV & 28 \\
\hline Site of metastases & \\
\hline liver & 18 \\
\hline lung & 5 \\
\hline peritoneum & 10 \\
\hline bone & 4 \\
\hline other & \\
\hline
\end{tabular}

2. Peripheral $C D 8^{+} P D-1^{+} T$ cells was related with expression of PD-L1 and the prognosis of patients with PDAC who received surgical resection.

Of the 177 patients received tumor resection, 67 tumor samples showed overexpression of PD-L1 and the representative IHC pictures were showed in figure 1A. Moreover, to determine the prognostic value of PDL1 expression level in PDAC, we used the Kaplan-Meier method and log-rank test to analyze the relationship between PD-L1 expression and patients' survival outcomes. We found that high PD-L1 expression in tumor tissues was significantly associated with short overall survival (supplemental figure 1). We further detected the peripheral blood T lymphocyte subtypes and found that 80 patients showed higher percentage of peripheral CD $8^{+} \mathrm{PD}-1^{+} \mathrm{T}$ cells and the representative pictures by flow cytometry were showed in figure 1B. Interestingly, we found that the percentage of peripheral CD8 ${ }^{+} \mathrm{PD}-1^{+} \mathrm{T}$ cells were significantly correlated with the PD-L1 expression in tumor tissues ( $r=0.541, p<0.001$, figure 1C). 
Moreover, the high CD8 ${ }^{+} \mathrm{PD}-1^{+} \mathrm{T}$ cells was significantly associated with short overall survival (figure 1D). We performed tracking test of peripheral blood $T$ lymphocyte subtypes for 30 months and found that in the high $\mathrm{CD} 8^{+} \mathrm{PD}-1^{+} \mathrm{T}$ cells, both the $\mathrm{CD} 3^{+} \mathrm{CD} 8^{+}$cells and $\mathrm{CD} 8^{+} \mathrm{PD}-1^{+}$cells were decreased and the changing status were showed in figure $1 \mathrm{E}, \mathrm{F}$. Therefore, we hypothesized that the CD $8^{+} \mathrm{PD}-1^{+}$cells were exhausted in the high CD8 $8^{+} \mathrm{PD}-1^{+} \mathrm{T}$ cells group which was related with PD-L1 expression. Restoring the percentage of $C D 8^{+} P D-1^{+} T$ cells and combined treated with anti-PD-1/PD-L1 could be promising treatments for patients with PDAC.

\section{Phenotypic analysis of peripheral blood T lymphocyte subtypes after ex vivo expansion.}

Mononuclear cells were harvested from peripheral blood before the treatment of ACT and expanded ex vivo. The total number of T cells was $2.57 \pm 1.06 * 10^{8}$ after 7 days, $28.1 \pm 6.38^{*} 10^{8}$ after 15 days, and $42.8 \pm 4.8^{*} 10^{8}$ after 30 days, respectively (figure $2 \mathrm{~A}, \mathrm{p}<0.001$. The $\mathrm{CD} 3^{+}, \mathrm{CD}^{+} \mathrm{CD} 4^{+}$and $\mathrm{CD} 3^{+} \mathrm{CD} 8^{+}$ lymphocytes increased significantly by day 15 compared with those of day 0 (figure $1 B, p<0.01$ ), but there was no significant difference between day 15 and 30 (figure $2 B, p>0.05$ ). $C D 8^{+} T$ cells exhibited enhanced expression of PD-1, LAG-3, and TIM-3 but not the costimulatory receptor 4-1BB after ex vivo expansion (Figure 2C). TIM-3 was the receptor most over-expressed by $\mathrm{CD}^{+}$cells after expansion for 15 days compared with the baseline expression, followed by PD-1 and LAG-3 $(15.2 \% \pm 3.6 \%, 10.4 \% \pm 3.2 \%$, and $5.3 \% \pm 2.3 \%$, respectively $(p<0.01)$ (Figure $2 \mathrm{C}$ ). The frequency of cells expressing these markers did not change from day 15-30. The cumulative frequency of T cells co-expressing at least 2 of these molecular markers was $9.6 \% \pm 3.8 \%$ of $C D 8^{+} T$ cells on day 15 , compared with $3.1 \% \pm 1.6 \%$ of $\mathrm{CD}^{+} \mathrm{T}$ cells on day 0 (Figure 2D). Figures 2E, F show the pattern of expression of these 4 receptors after ex vivo expansion for 15 days for a representative APC patient. This patient's $C D 8^{+} T$ cells displayed over-expression of PD-1, TIM-3, LAG-3, and 4-1BB.

\section{Increasing of $\mathrm{CD} 8{ }^{+} \mathrm{PD}-1^{+} \mathrm{T}$ cells and combined treatment with anti-PD-1 were related with favorable outcome in patients with APC.}

Of all the patients received ACT, 15 patients received combined treatment of anti-PD-1. The treatment methods were showed in figure $3 \mathrm{~A}, \mathrm{~B}$. The ratios of post treatment of ACT/pre-ACT CD8+PD-1+ T cells were detected and calculated. We performed ROC analysis to determine appropriate cut-off values. Survival analysis showed that patients with post/pre $>2$ of $C D 8^{+} P D-1^{+} T$ cells had a significantly favorable OS (median OS time 238 days versus 142 days, $P=0.024$, figure $3 C$ ) and progressive-free survival (PFS) (median PFS time 180 days vs 85 days, $P=0.002$, figure 3D). Moreover, patients with combined treatment of anti-PD-1 had significantly favorable both OS and PFS (figure 3E, F).

\section{5. $\mathrm{CD} 8^{+} \mathrm{PD}-1^{+} \mathrm{T}$ cells were tumor-reactive cells and could be applied for treatment of ACT.}

We isolated $\mathrm{CD} 8^{+}$(figure $4 \mathrm{~A}$ ) and $\mathrm{CD} 8^{+} \mathrm{PD}-1^{+} \mathrm{T}$ cells (figure 4B) from patient PBMC, expanded them in vitro for 15 days with IL-2, anti-CD3 stimulation, and irradiated feeders, and tested their ability to recognize autologous tumor cell lines by IFN-y-ELISPOT using HLA-A2+ tumor cell lines as target cells. 
Notably, CD8 ${ }^{+} \mathrm{PD}-1^{+} \mathrm{T}$ cells, but not $\mathrm{CD} 8^{+} \mathrm{PD}-1^{-} \mathrm{T}$ cells, contained the tumor-reactive cells as determined by IFN-y secretion and 4-1BB up-regulation after co-culture with the autologous tumor cell line (Figure 4C, D). Moreover, after expansion for 15 days, the IFN-y secretion and 4-1BB up-regulation were enhanced (Figure 4C, D). Additionally, CD $8^{+} \mathrm{PD}-1^{+} \mathrm{T}$ cells were capable of lysing the HLA-A2+ matched tumor cell lines and had stronger killing efficacy (Figure 4E). These data indicate that PD-1 expression identifies tumor-reactive peripheral blood $\mathrm{CD} 8^{+} \mathrm{T}$ cells, indicating that expression of PD-1 after ex vivo expansion may be used to prospectively identify and select a repertoire of $\mathrm{CD} 8^{+}$tumor-reactive cells.

\section{TCR diversity after expansion is associated with clinical outcomes.}

In order to calculate the TCR diversity of cultured T cells ex vivo, the Shannon diversity index ${ }^{26}$, TCR clonality ${ }^{27}$ and Evenness ${ }^{28}$ were used to characterize the diversity of TCR V $\beta$ CDR3 sequences of cultured $T$ cell samples of 19 of 32 patients from whom there was an adequate amount of specimen to performed CDR3 TCR V $\beta$ next generation sequencing. In light of the foregoing data demonstrating that CD8 ${ }^{+} \mathrm{PD}-1^{+} \mathrm{T}$ cells are tumor-reactive and their expansion correlated with the outcomes of patients treated with ACT and considering the role of the TCR repertoire in the anti-tumor response, we sought to assess the relationship between the $C D 8^{+} P D-1^{+} T$ cell frequency and the TCR repertoire. We first investigated the overlap in T cell clones in the 19 patients. As shown in Figure 5A, the unique TCR clones increased and the shared TCR clones decreased after $T$ cell expansion ex vivo in 10 patients $(P<0.05)$. However, as shown in Figure 5B, the unique TCR clones decreased and the shared TCR clones increased after T cell expansion ex vivo in 9 patients. Phenotypic analysis of PBMCs before the ACT treatment and at the end of the first cycle of therapy was performed. We observed that $\mathrm{CD}^{+}, \mathrm{CD}^{+} / \mathrm{CD} 4^{+}$and $\mathrm{CD}^{+} / \mathrm{CD} 8^{+}$cell subsets were significantly increased in the group in which TCR unique clones increased (UCI) (figure $5 \mathrm{C}, \mathrm{P}<0.05$ ) after treatment, while the $\mathrm{CD} 3^{+}, \mathrm{CD}^{+} / \mathrm{CD} 8^{+}$and $\mathrm{CD} 8^{+} / \mathrm{CD} 28^{-}$were significantly increased in the group of TCR unique clones decreased (UCD) (figure $5 C, P<0.05$ ) after treatment. Importantly, The $\mathrm{CD} 3^{+} / \mathrm{CD} 4^{+}$was significantly higher in the TCR UCl group compared with that of UCD group (figure $5 \mathrm{C}, \mathrm{P}<0.05$ ), and $\mathrm{CD} 8^{+} / \mathrm{CD} 28^{-}$were significantly lower in the $\mathrm{UCl}$ group compared with that of UCD group after treatment of ACT (figure $5 C, P<0.05$ ). Further survival analysis showed that the unique TCR clone increase was related to the prognosis of APC patients. Specifically, the analysis revealed a significantly favorable OS (median OS time 216 days versus 112 days, $P=0.031$, figure $5 \mathrm{D}$ ) and PFS (median PFS time 166 days vs 79 days, $P=0.043$, figure 5E) in patients in whom the unique TCR clones increased compared to those in whom unique TCR clones decreased. Finally, we performed correlation analysis to explore the relationship of $\mathrm{CD} 8^{+} \mathrm{PD}-1^{+} \mathrm{T}$ cell level and the TCR repertoire. The post/pre CD8 ${ }^{+} \mathrm{PD}-1^{+} \mathrm{T}$ cell ratio was significantly associated with the post/pre Shannon index (Figure 5F, $r^{2}=0.484, P=0.009$ ) and Clonality (Figure $5 F, r^{2}=0.579, P=0.002$ ), but not Evenness (Figure $5 F, r^{2}=0.018$, $\mathrm{P}=0.575)$. Moreover, the post/pre $\mathrm{CD} 8^{+} \mathrm{PD}-1^{+} \mathrm{T}$ cell ratio was significantly associated with an increase in unique TCR clones in patients who received treatment with ACT (Figure 5G, $r^{2}=0.464, P=0.001$ ).

\section{Cox proportional hazards analysis to identify the significant prognostic factors.}


Cox proportional hazards models were then used to quantify the prognostic signi[cance of risk factors after multivariable adjustment. A multivariable analysis was performed to assess the factors that demonstrated significant effects in univariate analysis. After adjusting for competing risk factors, post/pre $C D 8^{+} P D-1^{+} T$ cells $>2$ was an independent prognostic factor $\mathrm{OS}(\mathrm{P}=0.009)$ and PFS $(\mathrm{P}=0.012)$. The details are shown in supplemental figure 2.

\section{Discussion}

We previously observed improved outcome in patients with pancreatic cancer who received a combination of S-1 (which has demonstrated anti-tumor activity in pancreatic cancer) ${ }^{29}$ along with ACT immunotherapy, a cell product that includes dendritic, T, and NK-T cells. Because this is a heterogeneous population of cells, some of which have anti-tumor activity but some of which could be potentially immunosuppressive or cause toxicity, an effective biomarker that could specifically identify the repertoire of tumor-reactive and neoantigen-specific $\mathrm{CD} 8^{+} \mathrm{T}$ lymphocytes would be highly advantageous for enhancing clinical efficacy and safety ${ }^{30}$. In the present study, we found that among the bulk cell product expanded ex vivo, CD $8^{+} \mathrm{PD}-1^{+} \mathrm{T}$ cells could be identified as tumor-reactive and their expansion correlated with the breadth of TCR clonality and clinical outcome of patients treated with ACT.

PD-1 expression occurs in response to TCR signaling, and when PD-I binds to its ligands (PD-L1 or PDL2), it inhibits TCR/CD28 signaling and T-cell activation. Blockade of the PD-1 pathway reinvigorates exhausted T cells and can restore antitumor or antiviral immune responses ${ }^{31,32}$. Therefore, PD-1 expression is often thought of as immunosuppressive; however, T cells that up-regulate PD-1 are not always functional impaired or exhausted. In healthy donors, CD $8^{+} P D-1^{+} T$ cells from peripheral blood represent memory effector $T$ cells rather than dysfunctional $T$ cells ${ }^{33}$. In advanced melanoma patients, PD-1 is upregulated transiently and often sequentially by neoantigen-specific $\mathrm{CD} 8^{+} \mathrm{T}$ cells upon $\mathrm{T}$ cell activation and exposure to common gamma-chain cytokines including IL-2 in vitro ${ }^{22}$. Further, we found that $\mathrm{CD} 8^{+} \mathrm{PD}-1^{+} \mathrm{T}$ cells expanded in IL-2 were capable of secreting IFN- $\gamma$ and lysing tumor in vitro. This suggests that PD-1 may serve as a marker for the reproducible enrichment of tumor-reactive cells for patient treatment. Moreover, patients with post/pre CD $8^{+} \mathrm{PD}-1^{+} \mathrm{T}$ cell ratio $>2$ in the expanded product had significantly favorable OS and PFS compared with post/pre CD $8^{+} \mathrm{PD}-1^{+} \mathrm{T}$ cell ratio $\leq 2$. This supports the notion that immune dysfunction associated with co-expression of inhibitory receptors on $\mathrm{CD}^{+} \mathrm{T}$ cells can be reversed ${ }^{34,35}$ and that the robust expansion of $C D 8^{+} P D-1^{+} T$ cells may predict clinical benefit from ACT.

We also observed that TCR diversity may increase after expansion and is associated with outcomes in APC patients. The unique TCR clones increased and the shared TCR clones decreased after T cell expansion in vitro in 10 patients and these patients had favorable prognosis. Our results suggested that the expression of PD- 1 on $C D 8^{+} T$ cells captured the diverse repertoire of clonally expanded tumor- 
reactive lymphocytes and tumor-reactive clones might be highly expanded in the $\mathrm{CD} 8^{+}$population and preferentially expanded in the PD-1 ${ }^{+}$population.

CD28 is a co-stimulatory molecule which plays multiple roles in the activation, proliferation, and survival of $\mathrm{T}$ cells ${ }^{36,37}$. Accumulating evidence indicates that $\mathrm{CD} 8^{+} \mathrm{CD} 28^{-} \mathrm{T}$ cells are associated with inflammation-related disorders. Meanwhile, $\mathrm{CD} 8^{+} \mathrm{CD} 28^{-} \mathrm{T}$ cells are found in tumor microenvironments and the circulation of cancer patients. Both active and suppressive antitumor immune responses have been ascribed to $\mathrm{CD} 8^{+} \mathrm{CD} 28^{-} \mathrm{T}$ cell populations ${ }^{38,39}$. We found $\mathrm{CD} 8^{+} \mathrm{CD} 28^{-} \mathrm{T}$ cells were significantly increased after expansion in the group of patients with a decrease in unique TCR clones (UCD) after expansion and $C D 8^{+} C D 28^{-} T$ cells were significantly lower in the group with an increase in unique TCR clones (UCI). Further, TCR repertoire spectrum typing and sequencing was important for identifying whether $\mathrm{CD} 8^{+} \mathrm{CD} 28^{-} \mathrm{T}$ cells could recognize tumor antigens.

\section{Conclusion}

In summary, this study showed that the CD $8^{+} \mathrm{PD}-1^{+} \mathrm{T}$ cell subgroup was related with expression of PD-L1 and the prognosis of patients with PDAC who received surgical resection. The $\mathrm{CD} 8^{+} \mathrm{PD}-1^{+} \mathrm{T}$ cells were gradually exhausted and restoring it by treatment of ACT was associated with a significantly favorable OS and PFS. Moreover, combined ACT with anti-PD-1 was effective and promising in patients with APC. Further clinical trials are needed to verify these data.

\section{Abbreviations}

PDAC Pancreatic Ductal Adenocarcinoma

ACT adoptive T cell therapy

APC advanced pancreatic cancer

TCR T-cell receptor

PD-1 Programmed death receptor 1

OS overall survival

\section{Declarations}

\section{Ethics approval and consent to participate}

The study was approved by the Regional Ethical Review Board for Capital Medical University Cancer Center. All patients provided an informed written consent prior to study entry. 
All authors approved to publish this manuscript.

\section{Availability of data and materials}

The data used to support the findings of this study are available from the corresponding author upon request.

\section{Competing interests}

The authors who have taken part in this study declare that they have nothing to disclose regarding funding or conflict of interest with respect to this manuscript.

\section{Funding}

This work was supported by the National Natural Science Foundation of China (82002589) and the Program of Excellent Doctoral (Postdoctoral) of Zhongnan Hospital of Wuhan University (Grant No. ZNYB2019007).

\section{Authors' contributions}

Conceived and designed the experiments: Guoliang Qiao, Yufeng Yuan and Qian Zhu; Performed the experiments: Lefu Huang, Chang Xu, Deliang Guo, Shuo Wang, Jing Zhao, Yuguang Song, Bing Liu, Zheng Chen and Zhiyong Yang; Statistical analysis: Guoliang Qiao and Qian Zhu; Wrote the paper: Guoliang Qiao, Yufeng Yuan and Qian Zhu. All authors read and approved the final manuscript.

\section{Acknowledgements}

None.

\section{References}

1. Garrido-Laguna I, Hidalgo M. Pancreatic cancer: from state-of-the-art treatments to promising novel therapies. Nat Rev Clin Oncol 2015;12:319-34.

2. Sohn TA, Yeo CJ, Cameron JL, et al. Resected adenocarcinoma of the pancreas-616 patients: results, outcomes, and prognostic indicators. J Gastrointest Surg 2000;4:567-79.

3. Yabar CS, Winter JM. Pancreatic Cancer: A Review. Gastroenterol Clin North Am 2016;45:429-45.

4. Kleeff J, Korc M, Apte M, et al. Pancreatic cancer. Nat Rev Dis Primers 2016;2:16022.

5. Li W, Xu LP, L DIZ, et al. Cytokine-induced killer cell therapy for advanced pancreatic adenocarcinoma: A case report and review of the literature. Oncol Lett 2013;5:1427-1429.

6. Chung MJ, Park JY, Bang S, et al. Phase II clinical trial of ex vivo-expanded cytokine-induced killer cells therapy in advanced pancreatic cancer. Cancer Immunol Immunother 2014;63:939-46. 
7. Balachandran VP, Łuksza M, Zhao JN, et al. Identification of unique neoantigen qualities in long-term survivors of pancreatic cancer. Nature 2017;551:512-516.

8. Doi T, Muro K, Ishii H, et al. A Phase I Study of the Anti-CC Chemokine Receptor 4 Antibody, Mogamulizumab, in Combination with Nivolumab in Patients with Advanced or Metastatic Solid Tumors. Clin Cancer Res 2019;25:6614-6622.

9. Huber M, Brehm CU, Gress TM, et al. The Immune Microenvironment in Pancreatic Cancer. Int J Mol Sci 2020;21.

10. Topalian SL, Hodi FS, Brahmer JR, et al. Safety, activity, and immune correlates of anti-PD-1 antibody in cancer. N Engl J Med 2012;366:2443-54.

11. Sunshine J, Taube JM. PD-1/PD-L1 inhibitors. Curr Opin Pharmacol 2015;23:32-8.

12. Ho WJ, Jaffee EM, Zheng L. The tumour microenvironment in pancreatic cancer - clinical challenges and opportunities. Nat Rev Clin Oncol 2020;17:527-540.

13. Jiang N, Qiao G, Wang X, et al. Dendritic Cell/Cytokine-Induced Killer Cell Immunotherapy Combined with S-1 in Patients with Advanced Pancreatic Cancer: A Prospective Study. Clin Cancer Res 2017;23:5066-5073.

14. Hammerl D, Rieder D, Martens JWM, et al. Adoptive T Cell Therapy: New Avenues Leading to Safe Targets and Powerful Allies. Trends Immunol 2018;39:921-936.

15. Arasanz H, Gato-Canas M, Zuazo M, et al. PD1 signal transduction pathways in T cells. Oncotarget 2017;8:51936-51945.

16. Day CL, Kaufmann DE, Kiepiela P, et al. PD-1 expression on HIV-specific T cells is associated with Tcell exhaustion and disease progression. Nature 2006;443:350-4.

17. Trautmann L, Janbazian L, Chomont N, et al. Upregulation of PD-1 expression on HIV-specific CD8+ T cells leads to reversible immune dysfunction. Nat Med 2006;12:1198-202.

18. Ahmadzadeh $M$, Johnson $L A$, Heemskerk $B$, et al. Tumor antigen-specific CD8 $T$ cells infiltrating the tumor express high levels of PD-1 and are functionally impaired. Blood 2009;114:1537-44.

19. Varki V, loffe OB, Bentzen SM, et al. PD-L1, B7-H3, and PD-1 expression in immunocompetent vs. immunosuppressed patients with cutaneous squamous cell carcinoma. Cancer Immunol Immunother 2018;67:805-814.

20. Lu X, Yang L, Yao D, et al. Tumor antigen-specific CD8(+) T cells are negatively regulated by PD-1 and Tim-3 in human gastric cancer. Cell Immunol 2017;313:43-51.

21. Pasetto A, Gros A, Robbins PF, et al. Tumor- and Neoantigen-Reactive T-cell Receptors Can Be Identified Based on Their Frequency in Fresh Tumor. Cancer Immunol Res 2016;4:734-43.

22. Gros A, Robbins PF, Yao X, et al. PD-1 identifies the patient-specific CD8(+) tumor-reactive repertoire infiltrating human tumors. J Clin Invest 2014;124:2246-59.

23. Bailey P, Chang DK, Nones K, et al. Genomic analyses identify molecular subtypes of pancreatic cancer. Nature 2016;531:47-52. 
24. Ren J, Gwin WR, Zhou X, et al. Adaptive T cell responses induced by oncolytic Herpes Simplex Virusgranulocyte macrophage-colony-stimulating factor therapy expanded by dendritic cell and cytokineinduced killer cell adoptive therapy. Oncoimmunology 2017;6:e1264563.

25. EL K, P M. Nonparametric estimations from incomplete observations. J Am Stat Assoc 1958;53:457481.

26. Rempala GA, Seweryn M. Methods for diversity and overlap analysis in T-cell receptor populations. J Math Biol 2013;67:1339-68.

27. Qi Q, Liu Y, Cheng Y, et al. Diversity and clonal selection in the human T-cell repertoire. Proc Natl Acad Sci U S A 2014;111:13139-44.

28. Kvalseth TO. Evenness indices once again: critical analysis of properties. Springerplus 2015;4:232.

29. Ueno H, loka T, Ikeda M, et al. Randomized phase III study of gemcitabine plus S-1, S-1 alone, or gemcitabine alone in patients with locally advanced and metastatic pancreatic cancer in Japan and Taiwan: GEST study. J Clin Oncol 2013;31:1640-8.

30. Gros A, Parkhurst MR, Tran E, et al. Prospective identification of neoantigen-specific lymphocytes in the peripheral blood of melanoma patients. Nat Med 2016;22:433-8.

31. Parry RV, Chemnitz JM, Frauwirth KA, et al. CTLA-4 and PD-1 receptors inhibit T-cell activation by distinct mechanisms. Mol Cell Biol 2005;25:9543-53.

32. McKinney EF, Lee JC, Jayne DR, et al. T-cell exhaustion, co-stimulation and clinical outcome in autoimmunity and infection. Nature 2015;523:612-6.

33. Duraiswamy J, Ibegbu CC, Masopust D, et al. Phenotype, function, and gene expression profiles of programmed death-1(hi) CD8 T cells in healthy human adults. J Immunol 2011;186:4200-12.

34. Zippelius A, Batard P, Rubio-Godoy V, et al. Effector function of human tumor-specific CD8 T cells in melanoma lesions: a state of local functional tolerance. Cancer Res 2004;64:2865-73.

35. Wang SF, Fouquet $S$, Chapon $M$, et al. Early $T$ cell signalling is reversibly altered in PD-1+ T lymphocytes infiltrating human tumors. PLoS One 2011;6:e17621.

36. Dumitriu IE. The life (and death) of CD4+ CD28(null) T cells in inflammatory diseases. Immunology $2015 ; 146: 185-93$.

37. Maly K, Schirmer M. Corrigendum to "The Story of CD4 (+) CD28(-) T Cells Revisited: Solved or Still Ongoing?". J Immunol Res 2015;2015:251657.

38. Filaci G, Fenoglio D, Fravega $M$, et al. CD8+ CD28- T regulatory lymphocytes inhibiting T cell proliferative and cytotoxic functions infiltrate human cancers. J Immunol 2007;179:4323-34.

39. Casado JG, Soto R, DelaRosa O, et al. CD8 T cells expressing NK associated receptors are increased in melanoma patients and display an effector phenotype. Cancer Immunol Immunother 2005;54:1162-71.

\section{Figures}



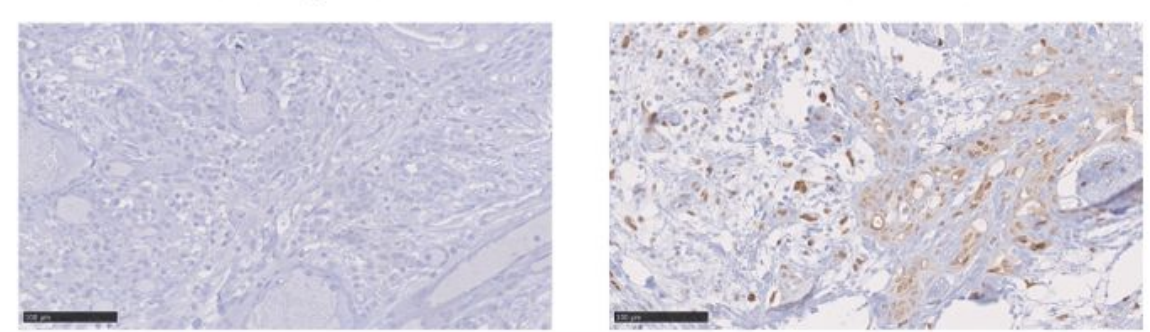

B
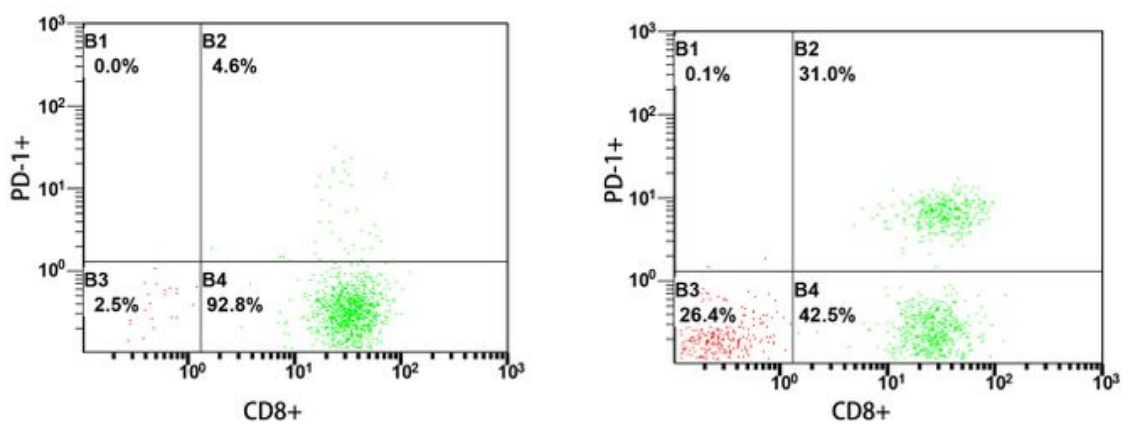

C

D
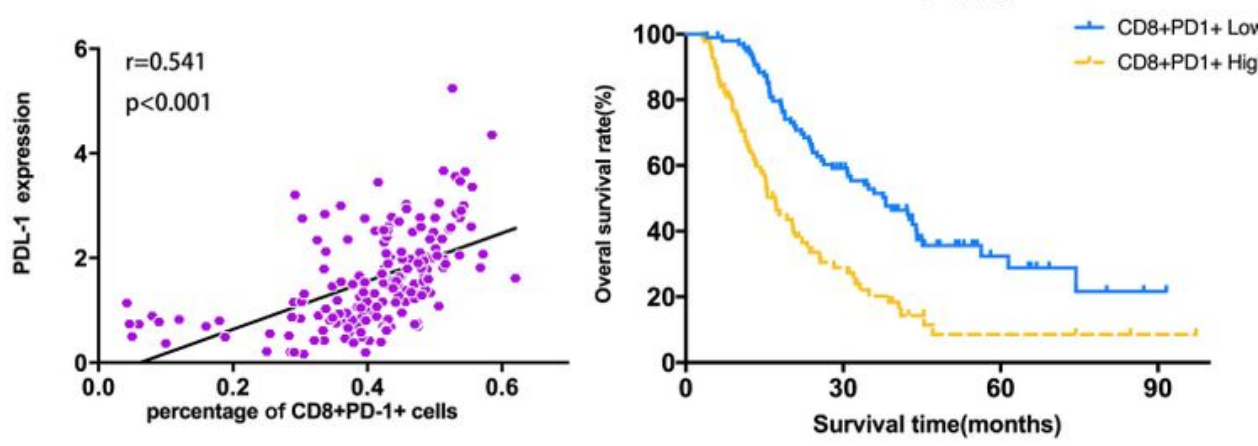

E

$\mathrm{CD} 3+\mathrm{CD} 8+$ cells

F
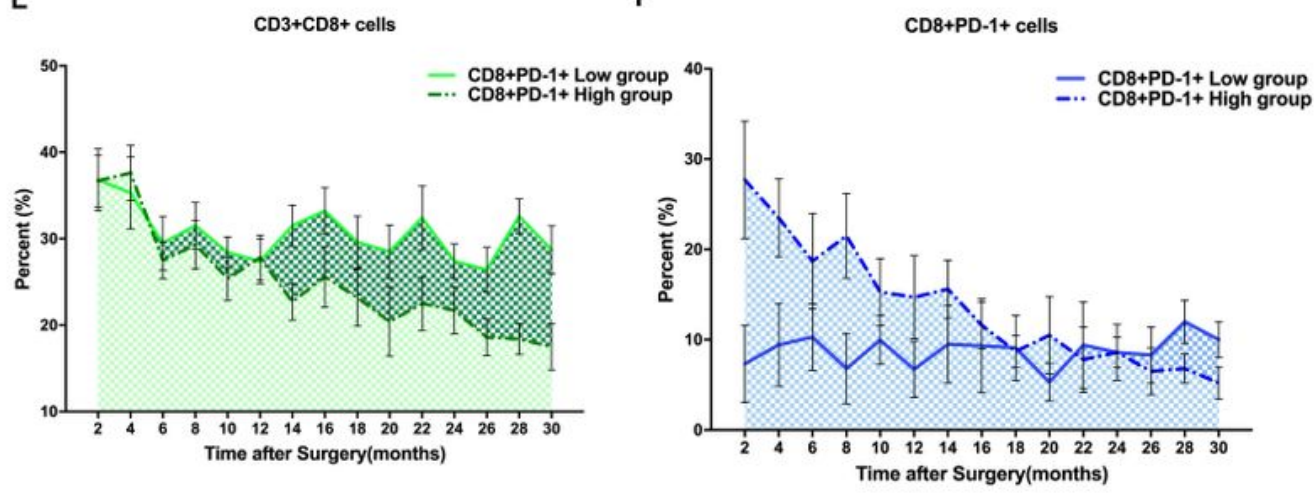

\section{Figure 1}

Peripheral CD8+PD-1+ T cells was related with expression of PD-L1 and the prognosis of patients with PDAC who received surgical resection. A: high expression vs. low expression of PD-L1 expression level in PDAC; $\mathrm{B}$ : the peripheral blood $\mathrm{T}$ lymphocyte subtypes detection and low peripheral CD8+PD-1+ T cells vs. high CD8+PD-1+ T cells detected by flow cytometry; $C$ : the percentage of peripheral CD8+PD-1+ T cells were significantly correlated with the PD-L1 expression in tumor tissues; D: the high CD8+PD-1+ T cells 
was significantly associated with short overall survival; E, F: tracking test of peripheral blood $T$ lymphocyte subtypes for CD3+CD8+ T cells and CD8+PD-1+ T cells for 30 months.

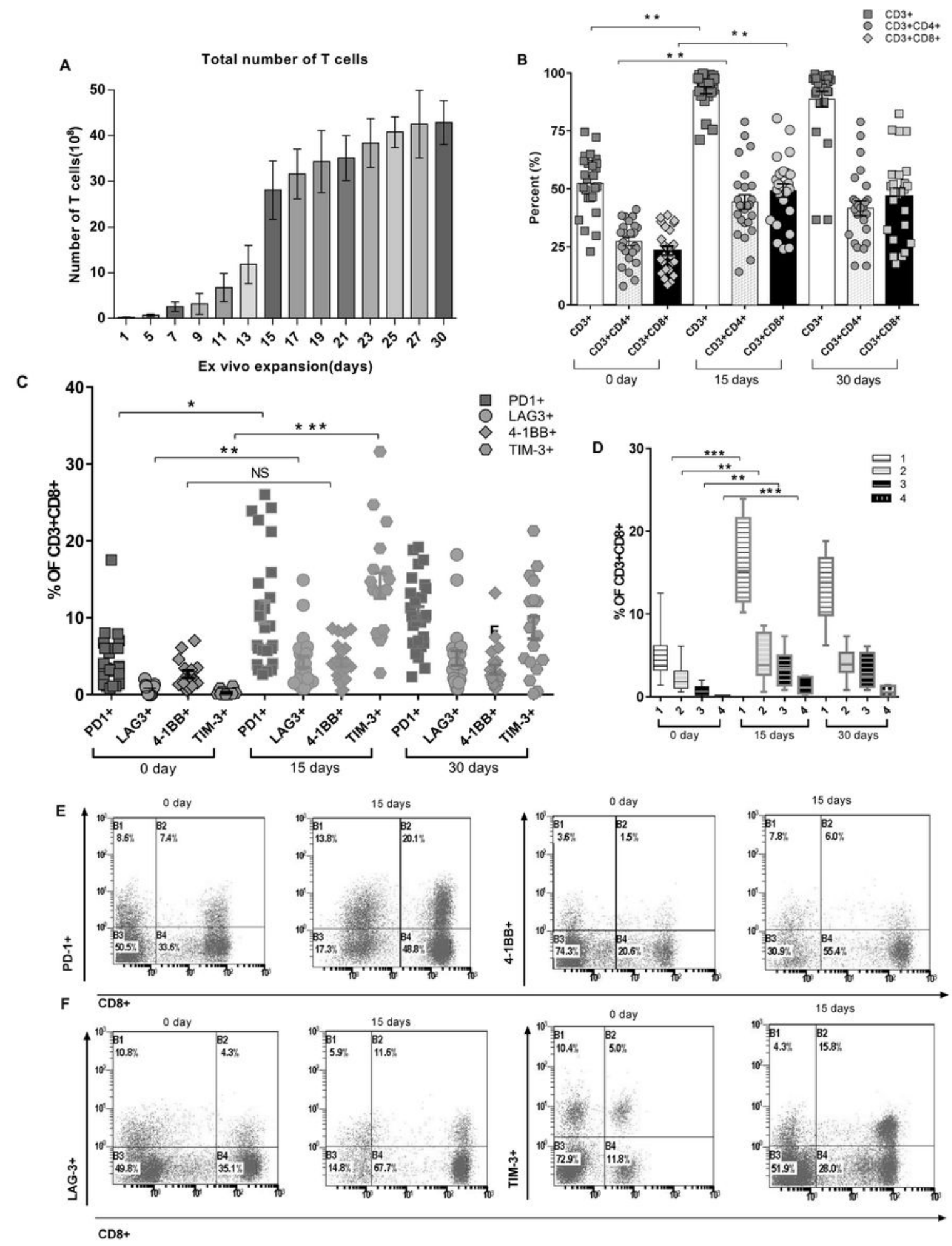

Figure 2

Quantitation of various phenotypic T cell proportions over time during the ex vivo PBMC expansion. A: total number of expanded $T$ cells daily from day 0 to day 30 . Each color represents $T$ cell assembly from day to day; B: Hierarchic phenotypic percentage of CD3+, CD3+CD4+ and CD3+CD8+ recorded at day 0, 
day 15 and day 30. Each individual actual fraction of $T$ cell subsets was recorded and shown as mean \pm SEM.; C: Functional stimulator and suppressor $T$ cell subsets of each individual were stratified during the continuous expansion at day 1, day 15 and day 30; D: co-expression percentage of PD-1, LAG-3, TIM-3, and 4-1BB in CD8+ PBMCs. The frequency of cells expressing either single or multiple markers above termed as 1, 2, 3, or 4 are shown. Bars represent maximum, minimum, and mean values; E: Fractioned sorting distributions of co-expression percentage of PD-1 and 4-1BB on CD8+ PBMCs through cytometric analysis (a representative patient is shown); F: Fractioned sorting distributions of co-expression percentage of LAG-3 and TIM-3 on CD8+ PBMCs through cytometric analysis (a representative patient is shown); ${ }^{P}<0.05,{ }^{*} \mathrm{P}<0.01,{ }^{\star * \star} \mathrm{P}<0.001$, Mann-Whitney test. 
A

\section{Treatment method 1}

Adoptive Cell Therapy (ACT)

Evaluation after two consecutive cycles of treatment
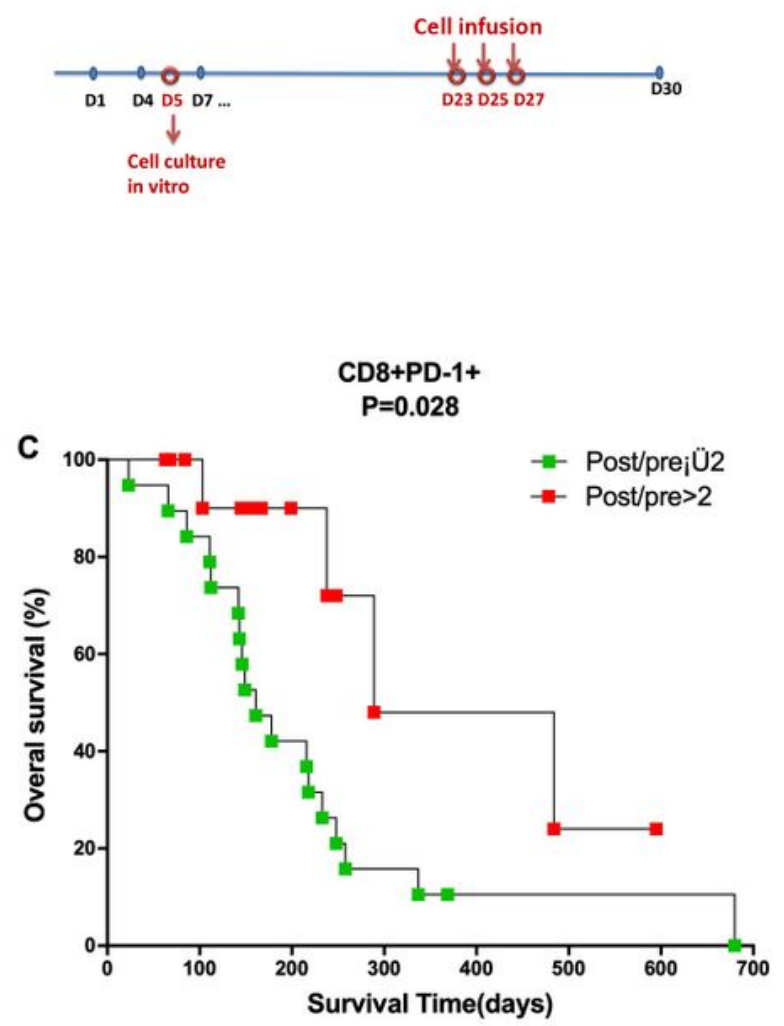

$P=0.019$

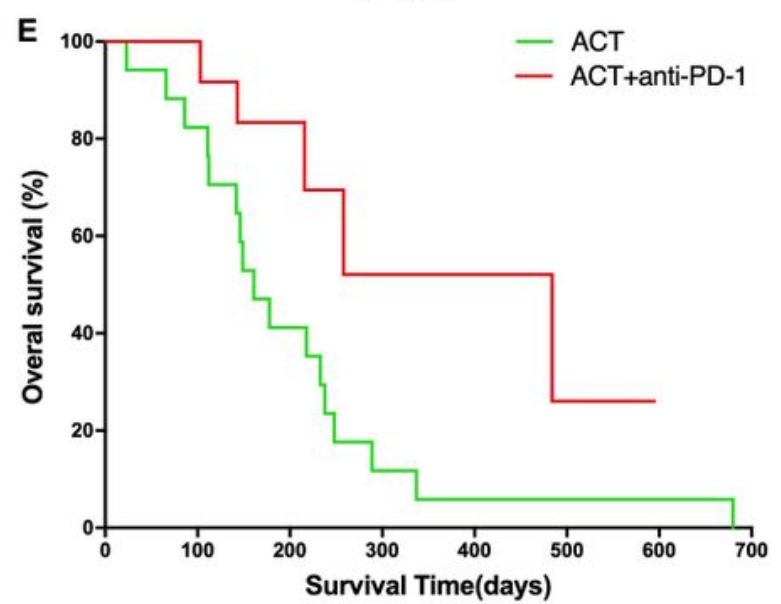

B

\section{Treatment method 2}

PD-1 inhibitor +ACT

Evaluation after two consecutive cycles of treatment

$$
\text { D8 }
$$

D8

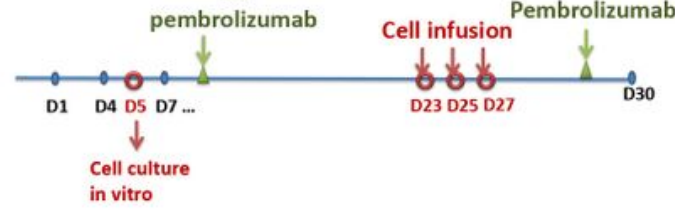

in vitro
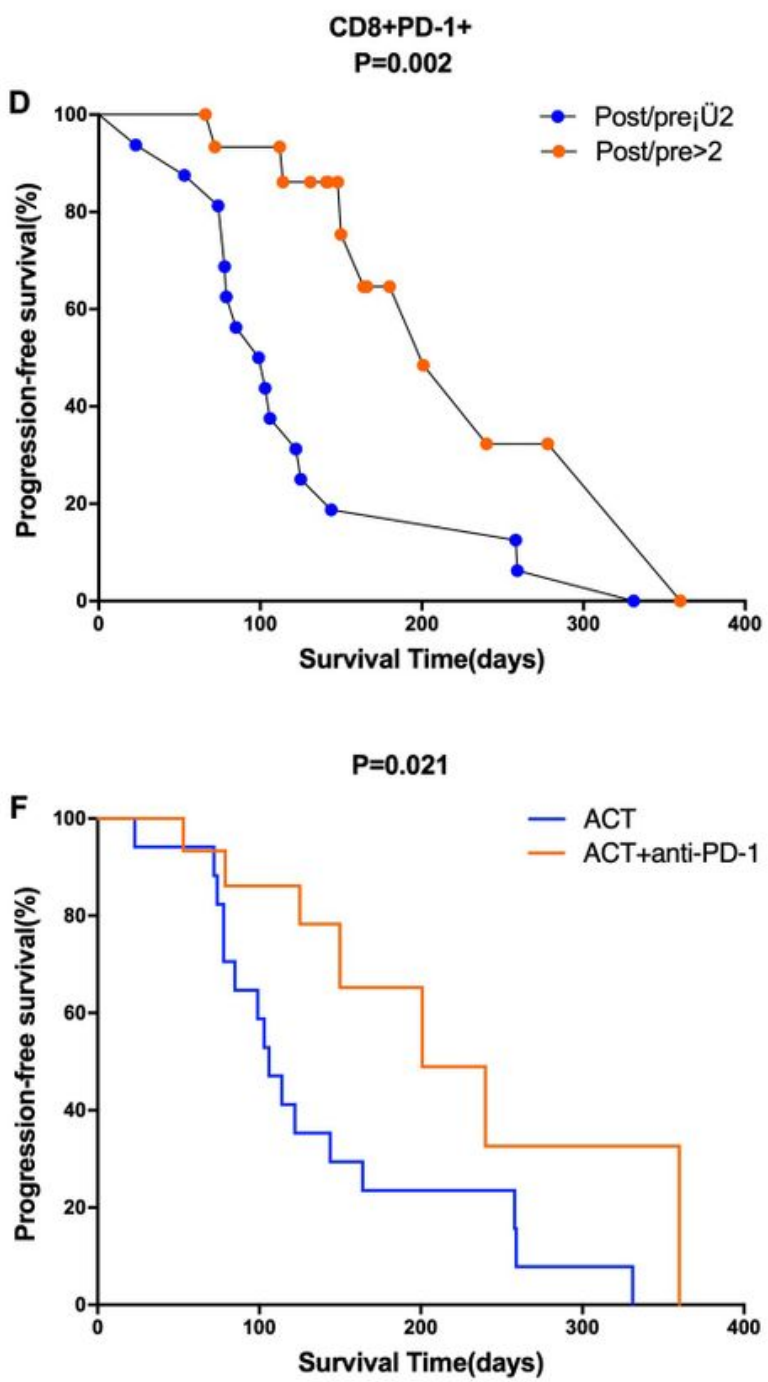

\section{Figure 3}

Survival analysis of subgroups divided by the treatment methods and level of CD8+PD- $1+T$ cells. A: treatment method of ACT in all patients with APC; B: treatment methods for patients received ACT combined with anti-PD-1; C, D: patients with post/pre>2 of CD8+PD-1+ T cells had significantly favorable OS and PFS compared with post/pre $\leq 2$ of CD8+PD- $1+T$ cells; E, F: patients received ACT combined with anti-PD-1 had favorable OS and PFS than patients received sole treatment of ACT. 


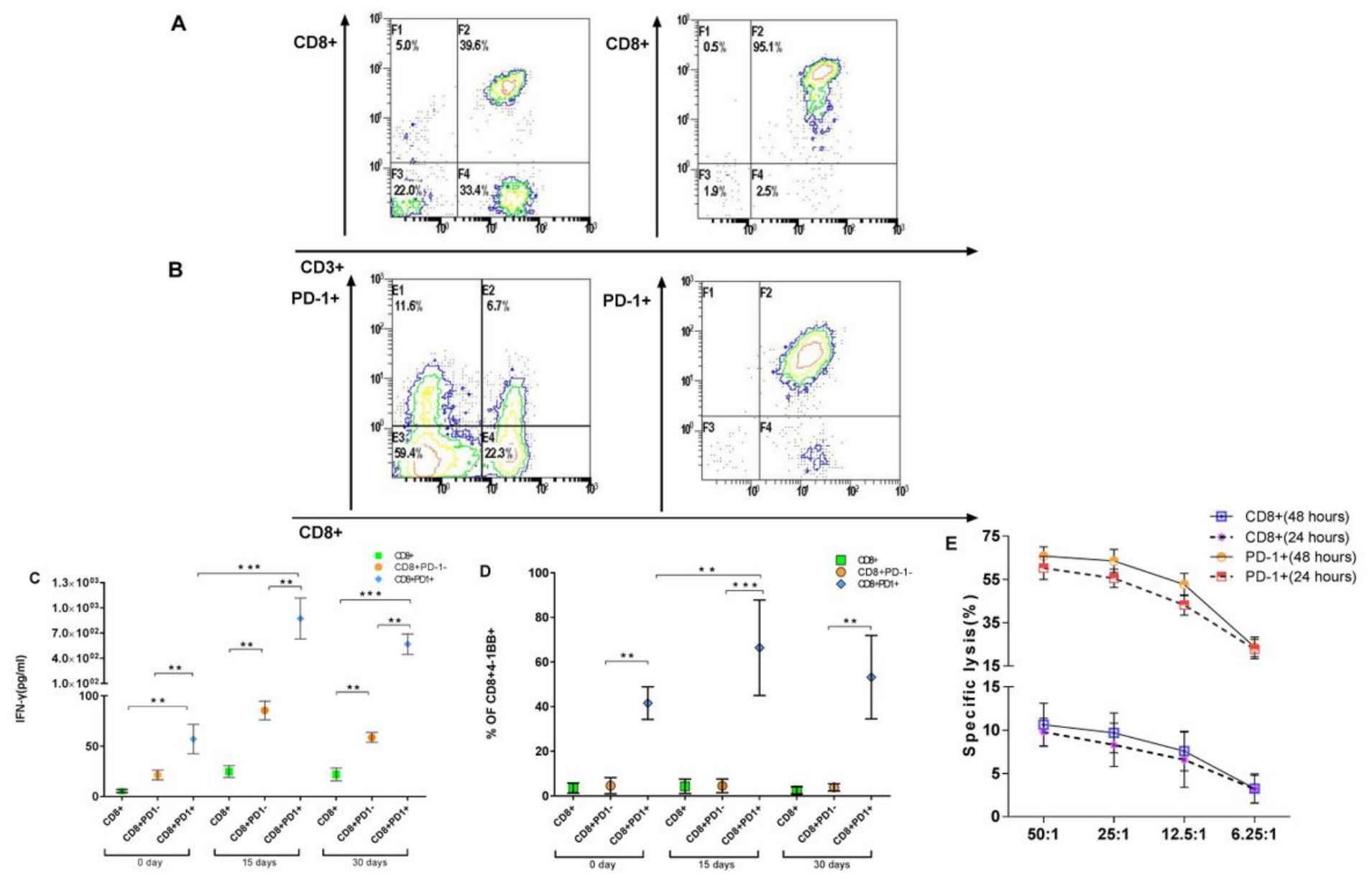

Figure 4

CD8+PD-1+ T cells could be identified as tumor-reactive CD8 T cells. A, B: CD8+ and CD8+PD-1+ T cells were sorted to identify the tumor-reactive T cells by ELISPOT and CCK-8; C, D: Reactivity of PD-1+ and PD$1-\mathrm{CD} 8+\mathrm{T}$ cells derived from patients against autologous tumor cell lines. IFN- $\gamma$ release and upregulation of 4-1BB (mean $\pm \mathrm{SD}$ ) are shown; E: Lysis of HLA-A2+ matched tumor cells by patient-derived CD8+ T cell. $\star P<0.05,{ }^{*} \mathrm{P}<0.01, * * * \mathrm{P}<0.001$, Mann-Whitney test. 

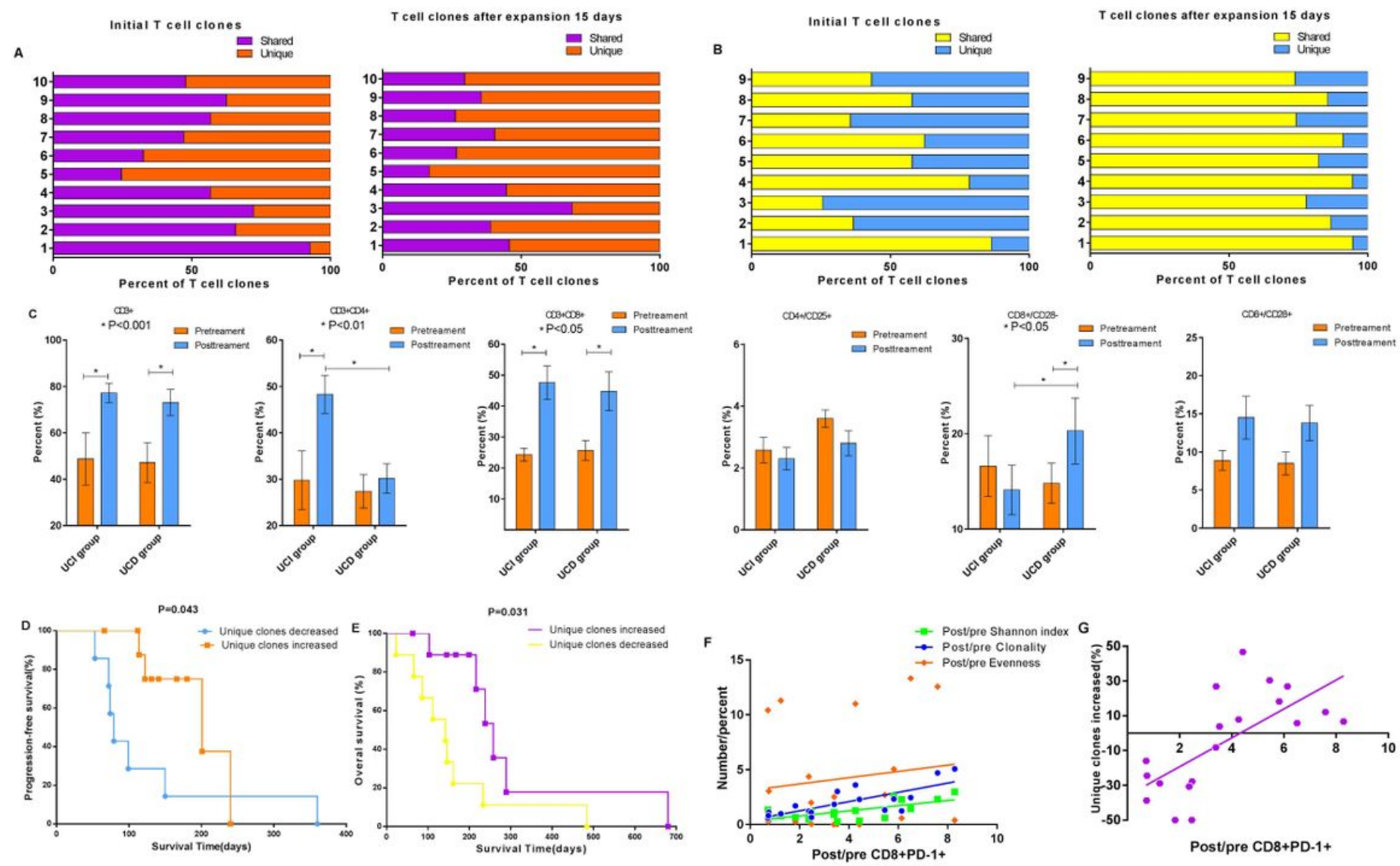

\section{Figure 5}

Changes in TCR subclones during ex vivo T cell expansion and the association of CD8+PD-1+ T cells with TCR diversity and subclones. A: the unique TCR clones increased and the shared TCR clones decreased after $T$ cell expansion ex vivo in 10 patients $(P<0.05)$; $B$ : the unique TCR clones decreased and the shared TCR clones increased after T cell expansion ex vivo in 9 patients; C: Peripheral blood T cell phenotype measurements via cytometry before and after the first cycle of ACT cell therapy divided by alteration of unique TCR clones; D, E: survival analysis showed that the group with increased unique TCR clones had significantly longer PFS $(p=0.043)$ and OS $(p=0.031)$; F: correlation analysis showed that the post/pre CD8+PD- $1+T$ cells were significantly associated with the post/pre Shannon index $(r 2=0.484, P=0.009)$ and Clonality ( $r 2=0.579, P=0.002)$, but not Evenness $(r 2=0.018, P=0.575) ; G$ : the post/pre CD8+PD- $1+T$ cells were significantly associated with the unique TCR repertoire clones in patients who received ACT $(\mathrm{r} 2=0.464, \mathrm{P}=0.001)$.

\section{Supplementary Files}

This is a list of supplementary files associated with this preprint. Click to download.

- Supplementalfigure1.jpg

- Supplementalfigure2.jpg 